\title{
Acknowledgement to Reviewers of Galaxies in 2016
}

\author{
Galaxies Editorial Office \\ Published: 10 January 2017 \\ MDPI AG, St. Alban-Anlage 66, 4052 Basel, Switzerland; galaxies@mdpi.com
}

The editors of Galaxies would like to express their sincere gratitude to the following reviewers for assessing manuscripts in 2016.

We greatly appreciate the contribution of expert reviewers, which is crucial to the journal's editorial process. We aim to recognize reviewer contributions through several mechanisms, of which the annual publication of reviewer names is one. Reviewers receive a voucher entitling them to a discount on their next MDPI publication and can download a certificate of recognition directly from our submission system. Additionally, reviewers can sign up to the service Publons (https://publons.com) to receive recognition. Of course, in these initiatives we are careful not to compromise reviewer confidentiality. Many reviewers see their work as a voluntary and often unseen part of their role as researchers. We are grateful to the time reviewers donate to our journals and the contribution they make.

If you are interested in becoming a reviewer for Galaxies, see the link at the bottom of the webpage http://www.mdpi.com/reviewers.

The following reviewed for Galaxies in 2016:

Abraham, Zulema
Agudo, Ivan
Alexandre, Jean
Aller, Hugh
Aller, Margo
Angelakis, Emmanouil
Asano, K.
Benbow, Wystan
Bhatta, Gopal
Boër, Michel
Böttcher, Markus
Bravetti, Alessandro
Brighenti, Fabrizio
Buchert, Thomas
Cassano, Rossella
Chavanis, Pierre-Henri
Cheung, Teddy
Contopoulos, Ioannis
Corda, Christian
D'Onghia, Elena
D'Ammando, Filippo
Das, Saurya
D'Avanzo, Paolo
Dvorak, Rudolf
Eckert, Dominique
Famaey, Benoit
Fan, Junhui

\author{
Fromm, Christian \\ Gabuzda, Denise \\ Galbany, Lluis \\ Gastaldello, Fabio \\ Gaur, Haritma \\ Gear, Walter \\ Gentile, Gianfranco \\ Ghisellini, Gabriele \\ Gianfranco, Brunetti \\ Gielen, Steffen \\ Giroletti, Marcello \\ Gómez, Jose L. \\ Grøn, Øyvind \\ Guidorzi, Cristiano \\ Guo, Fulai \\ Hada, Kazuhiro \\ Hikage, Chiaki \\ Homan, Dan \\ Johnson, Michael D. \\ Jorstad, Svetlana \\ Kasai, Masumi \\ Kovalev, Yuri \\ Krichbaum, Thomas \\ Kroupa, Pavel \\ Kunz, J. \\ L'Huillier, Benjamin \\ Laing, Robert
}

\author{
Larionov, Valeri \\ Leclercq, Florent \\ Lee, Chung-Chi \\ Lico, Rocco \\ Lister, Matt \\ Liu, Tong \\ Luongo, Orlando \\ Lyutikov, Maxim \\ Mann, Robert B. \\ Mapelli, Michela \\ Marscher, Alan \\ Marti, J. M. \\ Mastichiadis, Apostolos \\ Matos, T. \\ Meis, Constantin \\ Meneghetti, Massimo \\ Meyer, Eileen \\ Migliori, Giulia \\ Miller, Hugh R. \\ Moscardini, Lauro \\ Mukherjee, Reshmi \\ Nalewajko, Krzysztof \\ Nipoti, C. \\ Noble, Scott \\ Nojiri, Shin'ichi \\ Paneque, David \\ Pearson, Timothy
}


Piattella, Oliver Fabio Piner, Glenn

Pozanenko, Alexei Pushkarev, Alexander Ramakrishnan, Venkatessh Rasia, Elena Rodrigues, Davi Saavedra, Joel Savolainen, Tuomas.k. Shigeyama, Toshikazu
Sikora, Marek

Simon, Joan

Smith, Paul

Sun, Hui

Tanaka, Takahiro

Tchekhovskoy, Alexander

Tollerud, Erik J.

Trigo-Rodriguez, Josep M.

Ucci, Graziano

Valentino, Eleonora Di

\author{
Vazza, Franco \\ Venturi, Tiziana \\ Walker, Craig \\ Wang, Xiang-Yu \\ Wardle, John \\ Webb, James R. \\ $\mathrm{Wu}$, Xue-Feng \\ $\mathrm{Yu}$, Hoi-Fung \\ Yu, Yun-Wei \\ Zhang, Bing
}

(C) 2017 by the authors; licensee MDPI, Basel, Switzerland. This article is an open access article distributed under the terms and conditions of the Creative Commons Attribution (CC-BY) license (http://creativecommons.org/licenses/by/4.0/). 